\title{
OP-0043 - Cost-effectiveness analysis for early diagnosis of diabetes compared with non-early diagnosis
}

\section{Limin Wang, MS; Mei Zhang, PhD; Zhenping Zhao, MS; Xiao Zhang, PhD; Zhengjing \\ Huang; Linhong Wang, PhD, National Center for Chronic and Non-communicable Disease Control and Prevention, Chinese Center For Disease Control and Prevention, Beijing, China.}

\begin{abstract}
Background
Diabetes has been one of the major public health challenges in China with prevalence over $10 \%$ for population above 18 years old, and over $60 \%$ of them were unware of the disease until late stage with perceptible symptoms or even complications. It will cause significant disease burden to patients and healthcare system. Early diagnosis of diabetes, and consequently early intervention, are valuable and important.
\end{abstract}

\section{Aim}

The purpose of this study is to perform cost-effectiveness analysis comparing early diagnosis of diabetes with non-early diagnosis, to demonstrate long term cost and health outcomes of early and non-early diagnosis.

\section{Method}

Based on demographic and medical data obtained from China chronic disease risk factor surveillance (2013) among 170287 residents, a simulated cohort of 1000 early diagnosed cases and 1000 non-early diagnosed cases was set up. 30-year incidence of complications, costs, and outcomes were predicted with CORE diabetes model ${ }^{1}$. Costs included cost of complications and management, and outcomes included life years and QALYs (quality-adjusted life-years). Student's t test was performed for comparison of early and non-early diagnosis group. ICER (incremental cost-effectiveness ratio) with cost and QALYs was calculated with 3\% discount rate and one-way analysis for major parameters was performed to demonstrate the robustness of the result.

\section{Results}

Compared with non-early diagnosis group, early diagnosis group had lower cardiovascular death incidence (Figure 1) and greater life expectancy (14.41 years vs. 14.11 years). The total costs were CNY 152,313 and 160,678 for early and non-early diagnosis group respectively, and the QALYs gained were 10.26 and 10.00 respectively. Early diagnosis was dominant on non-early diagnosis with lower cost and better outcomes (Figure 2), and one-way sensitivity analysis demonstrated the robustness of the result (Table 1)

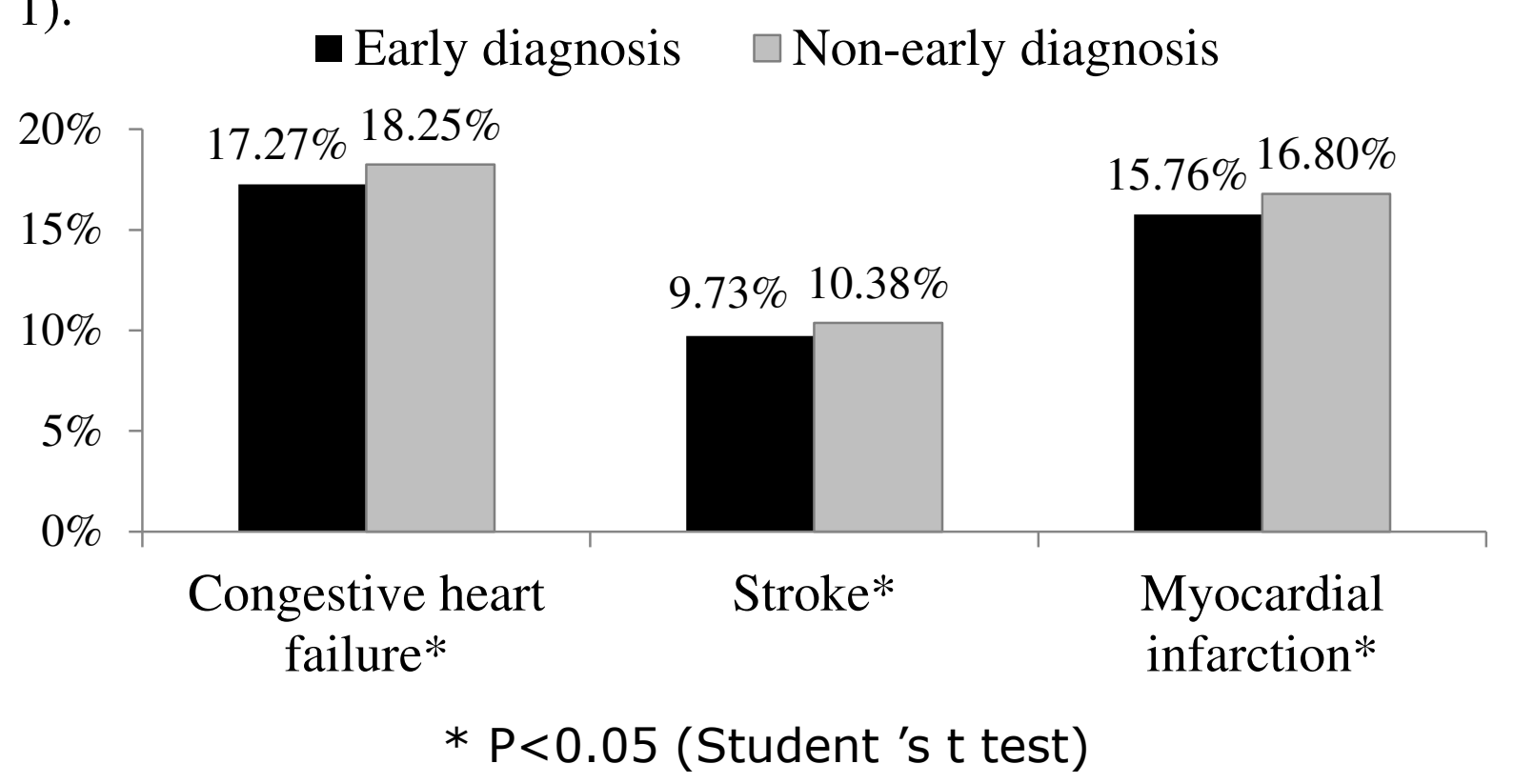

Figure 1 Reduction in cumulative incidence $(\%)$ of cardiovascular death for early diagnosis of diabetes compared with non-early diagnosis

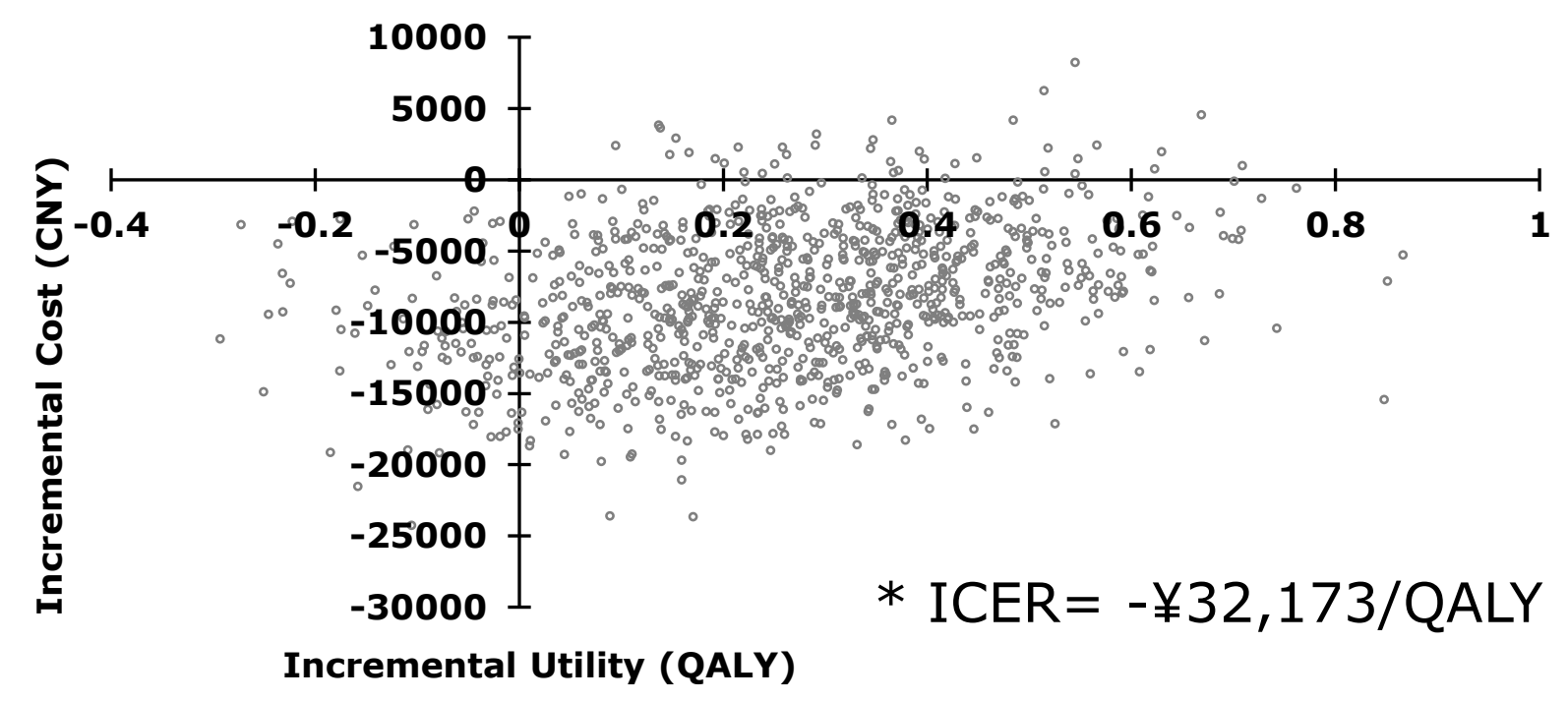

Figure 2 Cost-effectiveness plane of ICER for the early diagnosis of diabetes compared with non-early diagnosis

\section{Discussion}

This study demonstrated the advantages of early diagnosis of diabetes for medical cost saving and better long-term outcomes. This study was conducted with data obtained from a cross-sectional study in China and prediction of CORE model which was developed in western countries. Longitudinal long term study in China should be conducted to generate more solid evidence for validation of results of this study.

Ref: 1. Palmer et al. Curr Med Res Opin 2004; 20: S5-26.

Table 1 Sensitivity analyses of cost-effectiveness of diabetes early treatment

\begin{tabular}{|c|c|c|c|c|c|c|c|}
\hline & \multicolumn{3}{|c|}{ Quality-adjusted life expectancy(QALY) } & \multicolumn{3}{|c|}{ Direct costs $(\mathrm{CNY})$} & \multirow{2}{*}{$\begin{array}{c}\text { ICER } \\
\Delta \operatorname{costs} / \triangle \mathrm{QALY}\end{array}$} \\
\hline & IDeg & IGlar & $\triangle \mathrm{QALY}$ & IDeg & IGlar & $\Delta$ costs & \\
\hline 5-year time horizon & 3.17 & 3.16 & 0.01 & 36155.61 & 36854.32 & -698.71 & -145565 \\
\hline 10-year time horizon & 5.62 & 5.60 & 0.02 & 68192.35 & 70669.97 & -2477.62 & -102806 \\
\hline 20-year time horizon & 8.79 & 8.66 & 0.13 & 119158.36 & 125788.51 & -6630.15 & -52956 \\
\hline 30-year time horizon (original) & 10.26 & 10.00 & 0.26 & 152313.48 & 160678.44 & -8364.95 & -32148 \\
\hline Discounting rate $0 \%$ & 14.55 & 14.08 & 0.48 & 229693.59 & 242879.63 & -13186.03 & -27748 \\
\hline Discounting rate $5 \%$ & 8.40 & 8.22 & 0.18 & 120323.34 & 126666.15 & -6342.81 & -35297 \\
\hline complication costs increased by $20 \%$ & 10.26 & 10.00 & 0.26 & 174066.30 & 184251.08 & -10184.79 & -39142 \\
\hline complication costs decreased by $20 \%$ & 10.26 & 10.00 & 0.26 & 130560.64 & 137105.75 & -6545.11 & -25154 \\
\hline
\end{tabular}

